\title{
Copious Amounts of Dust and Gas in a $z=7.5$ Quasar Host Galaxy
}

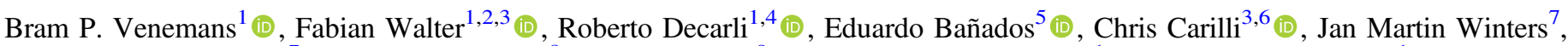 \\ Karl Schuster $^{7}$, Elisabete da Cunha $^{8}(\mathbb{D})$, Xiaohui Fan ${ }^{9}$ (D), Emanuele Paolo Farina ${ }^{1}$ (1) , Chiara Mazzucchelli ${ }^{1}(\mathbb{D}$, \\ Hans-Walter Rix ${ }^{1}$ (D), and Axel Weiss ${ }^{10}$ (D) \\ ${ }^{1}$ Max-Planck Institute for Astronomy, Königstuhl 17, D-69117 Heidelberg, Germany; venemans@mpia.de \\ 2 Astronomy Department, California Institute of Technology, MC105-24, Pasadena, CA 91125, USA \\ ${ }^{3}$ National Radio Astronomy Observatory, Pete V. Domenici Array Science Center, P.O. Box 0, Socorro, NM 87801, USA \\ ${ }^{4}$ Osservatorio Astronomico di Bologna, via Gobetti 93/3, I-40129 Bologna, Italy \\ ${ }^{5}$ The Observatories of the Carnegie Institution for Science, 813 Santa Barbara Street, Pasadena, CA 91101, USA \\ ${ }^{6}$ Astrophysics Group, Cavendish Laboratory, JJ Thomson Avenue, Cambridge CB3 OHE, UK \\ ${ }^{7}$ Institut de Radioastronomie Millimétrique (IRAM), 300 rue de la Piscine, F-38406 Saint Martin d'Hères, France \\ ${ }^{8}$ Research School of Astronomy and Astrophysics, Australian National University, Canberra, ACT 2611, Australia \\ ${ }^{9}$ Steward Observatory, The University of Arizona, 933 North Cherry Avenue, Tucson, AZ 85721-0065, USA \\ ${ }^{10}$ Max-Planck-Institut für Radioastronomie, Auf dem Hügel 69, D-53121 Bonn, Germany \\ Received 2017 August 29; revised 2017 October 13; accepted 2017 October 17; published 2017 December 6
}

\begin{abstract}
We present IRAM/NOEMA and JVLA observations of the quasar J1342+0928 at $z=7.54$ and report detections of copious amounts of dust and [C II] emission in the interstellar medium (ISM) of its host galaxy. At this redshift, the age of the universe is $690 \mathrm{Myr}$, about $10 \%$ younger than the redshift of the previous quasar record holder. Yet, the ISM of this new quasar host galaxy is significantly enriched by metals, as evidenced by the detection of the [C II] $158 \mu \mathrm{m}$ cooling line and the underlying far-infrared (FIR) dust continuum emission. To the first order, the FIR properties of this quasar host are similar to those found at a slightly lower redshift $(z \sim 6)$, making this source by far the FIR-brightest galaxy known at $z \gtrsim 7.5$. The [C II] emission is spatially unresolved, with an upper limit on the diameter of $7 \mathrm{kpc}$. Together with the measured FWHM of the [C II] line, this yields a dynamical mass of the host of $<1.5 \times 10^{11} M_{\odot}$. Using standard assumptions about the dust temperature and emissivity, the NOEMA measurements give a dust mass of (0.6-4.3) $\times 10^{8} M_{\odot}$. The brightness of the [C II] luminosity, together with the high dust mass, imply active ongoing star formation in the quasar host. Using [C II]-SFR scaling relations, we derive star formation rates of $85-545 M_{\odot} \mathrm{yr}^{-1}$ in the host, consistent with the values derived from the dust continuum. Indeed, an episode of such past high star formation is needed to explain the presence of $\sim 10^{8} M_{\odot}$ of dust implied by the observations.
\end{abstract}

Key words: cosmology: observations - galaxies: active - galaxies: high-redshift - galaxies: ISM

\section{Introduction}

The advent of large, wide-area optical and infrared surveys has resulted in the discovery of luminous quasars out to the highest redshifts, $z \gtrsim 7$ (e.g., Fan et al. 2006; Venemans et al. 2013; Bañados et al. 2016; Mazzucchelli et al. 2017). These quasars are powered by supermassive, $\sim 10^{9} M_{\odot}$ black holes that accrete near the Eddington limit (e.g., De Rosa et al. 2014; Mazzucchelli et al. 2017). Since their discovery, the presence of such massive black holes has been a puzzle, as they require either very efficient accretion mechanisms from stellar black hole seeds $\left(\sim 100 M_{\odot}\right.$; e.g., Volonteri 2012$)$ or the formation of massive seeds, e.g., via direct gas collapse ( $\sim 10^{3-5} M_{\odot}$; e.g., Agarwal et al. 2012; Regan et al. 2017).

Likewise, studies of the host galaxies of these distant quasars have revealed the presence of large amounts of dust and gas out to $z \sim 7$ (e.g., Bertoldi et al. 2003; Walter et al. 2003; Maiolino et al. 2005; Venemans et al. 2012, 2016; Wang et al. 2013; Willott et al. 2015). The associated molecular gas masses are $>10^{10} M_{\odot}$ and provide the fuel for long-lasting $\left(>10^{7-8}\right.$ years $)$ episodes of ultraluminous infrared galaxy (ULIRG)-like star formation (with star formation rates (SFRs) $\sim 100-1000 M_{\odot} \mathrm{yr}^{-1}$ ).

The observed tracers ([C II], [C I], CO, far-infrared (FIR) continuum) require that the interstellar medium (ISM) of the host galaxies is chemically enriched. While metal-enriched material is also evident from broad emission lines in the quasar's rest-frame UV spectrum (e.g., Jiang et al. 2007; De
Rosa et al. 2014), these emission lines originate from a concentrated region $(\ll 1 \mathrm{pc})$ around the black hole, the so-called broad-line region (BLR). However, since the BLR total mass is only $10^{4-5} M_{\odot}$ (e.g., Ferland 2004), a modest amount of metals, $\sim 10^{3-4} M_{\odot}$, can explain the observed high BLR metallicities $\left(Z \sim 10 Z_{\odot}\right.$; e.g., Dietrich et al. 2003; Juarez et al. 2009). On the other hand, the enrichment of the quasar host is on significantly larger scales ( $\sim \mathrm{kpc}$; e.g., Walter et al. 2009; Wang et al. 2013; Venemans et al. 2016), which requires a galaxy-wide chemical enrichment due to extended star formation. The enrichment on galactic scales seen in dust and gas thus puts constraints on early metal production in the quasar host (e.g., Michałowski et al. 2010; Gall et al. 2011b). Star formation is only indirectly traced by the FIR emission, and to date the stellar component of the quasar host remains elusive (e.g., Decarli et al. 2012).

To further constrain the formation of dust and enrichment of gas in the ISM in the earliest galaxies, studies need to be pushed back in time, i.e., to the highest possible redshifts. The highest-redshift quasar in which gas and dust have been detected to date is $\mathrm{J} 1120+0641$ (Mortlock et al. 2011) at a redshift of $z=7.09$ (Venemans et al. 2017). Here, we report the detection of gas and dust emission in a newly discovered quasar at $z=7.5, \mathrm{~J} 1342+0928$ (Bañados et al. 2017). The redshift of the quasar derived from the $\mathrm{Mg}$ II line is $z_{\mathrm{Mg} \mathrm{II}}=7.527 \pm 0.004$ (age of the universe: $690 \mathrm{Myr}$ ). From 
the width of the $\mathrm{Mg}$ II line and the strength of the continuum, Bañados et al. (2017) estimate that the quasar is powered by accretion onto a $7.8_{-1.9}^{+3.3} \times 10^{8} M_{\odot}$ black hole. The quasar has an absolute magnitude of $M_{1450} \AA=-26.8$ and shares many of the physical properties seen in quasars observed at $z \sim 6-7$ (Bañados et al. 2017).

Throughout this Letter, we adopt a concordance cosmology with $\Omega_{M}=0.3, \Omega_{\Lambda}=0.7$, and $H_{0}=70 \mathrm{~km} \mathrm{~s}^{-1} \mathrm{Mpc}^{-1}$. The physical scale at $z=7.54$ is $5.0 \mathrm{kpc} \operatorname{arcsec}^{-1}$. All magnitudes are on the $\mathrm{AB}$ system.

\section{Observations}

\subsection{NOEMA Observations}

$[\mathrm{C} \mathrm{II}]_{3 / 2-1 / 2} 158 \mu \mathrm{m}$ (hereafter [C II]), $\mathrm{CO}(7-6), \mathrm{CO}(10-9)$, $\mathrm{H}_{2} \mathrm{O}$, and $[\mathrm{C} \mathrm{I}]_{2-1}$ observations of $\mathrm{J} 1342+0928$ were performed with the IRAM NOrthern Extended Millimeter Array (NOEMA). Observations were done with the array in compact configuration, using 7-8 antennas. All of the NOEMA data have been reduced using the latest version of the GILDAS software. ${ }^{11}$

The observations were gathered between 2017 March 15 and May 21 in various visits. For the [C II] observations, the NOEMA receiver $3(1.2 \mathrm{~mm})$ was tuned to $224.121 \mathrm{GHz}$ in the first execution, and to $222.500 \mathrm{GHz}$ in all the other visits, in order to better encompass the line within the WideX $3.6 \mathrm{GHz}$ bandwidth. The $\mathrm{CO}(10-9)$ line and the $\mathrm{H}_{2} \mathrm{O} 3(2,1)-3(1,2)$ line at rest-frequency $1162.91 \mathrm{GHz}$ were observed in a single frequency setting, with NOEMA receiver $2(2 \mathrm{~mm})$ tuned to 135.495 GHz. The $\mathrm{CO}(7-6)$ and $[\mathrm{C}]_{2-1}$ lines were observed with the $3 \mathrm{~mm}$ receivers tuned to $94.587 \mathrm{GHz}$. The radio quasar $1345+125$ served as amplitude and phase calibrator. Additional calibrators used in the bandpass calibration included 3C273 and 3C454.3. The star MWC 349 was used to set the absolute flux scale. Measured line fluxes and continuum flux densities in Section 3 and Table 1 only include statistical errors and do not take the systematic flux calibration uncertainties of $\sim 10 \%$ into account. The total integration time on-source was 13.6, 3.8, and $11.1 \mathrm{hr}$ (8 antenna equivalent) in the $1 \mathrm{~mm}$, $2 \mathrm{~mm}$, and $3 \mathrm{~mm}$ bands, respectively. Imaging was performed using natural weighting, in order to maximize sensitivity. The resulting synthesized beams are 2 !" $4 \times 1$ " 5,3 " $6 \times 2$ ". 5 , and $5 . .8 \times 3 . " 4$ and the final $1 \mathrm{~mm}, 2 \mathrm{~mm}$, and $3 \mathrm{~mm}$ cubes reach a sensitivity of $0.47 \mathrm{mJy}_{\text {beam }}^{-1}, 0.41 \mathrm{mJy}^{-1}$ beam $^{-1}$, and $0.17 \mathrm{mJy}$ beam $^{-1}$ per $100 \mathrm{~km} \mathrm{~s}^{-1}$ channel $(1-\sigma)$, respectively. In the $1 \mathrm{~mm}$ cube, both the [C II] emission and the underlying dust continuum are significantly detected (Figure 1 and Section 3), while no emission was detected in the other two cubes.

In the continuum images, an additional source is located $\sim 10^{\prime \prime}$ northeast of the quasar (see Figure 2) with flux densities of $S_{223.5 \mathrm{GHz}}=434 \pm 73 \mu \mathrm{Jy}, S_{135.5 \mathrm{GHz}}=197 \pm 46 \mu \mathrm{Jy}$, and $S_{95 \mathrm{GHz}}=41 \pm 16 \mu \mathrm{Jy}$. The spectrum of this object does not show emission lines. While the redshift remains unknown, the lack of line emission in the $1 \mathrm{~mm}$ datacube, which covers a [C II] redshift of $\Delta z \approx 0.1$ around that of the quasar, makes it unlikely that this source is physically associated with J1342+0928.

\footnotetext{
${ }^{11}$ http://www.iram.fr/IRAMFR/GILDAS
}

Table 1

Observed and Derived Properties of J1342+0928

\begin{tabular}{|c|c|}
\hline$\overline{\text { R.A. (J2000) }}$ & $13^{\mathrm{h}} 42^{\mathrm{m}} 08^{\mathrm{s}} .097$ \\
\hline Decl. (J2000) & $+09^{\circ} 28^{\prime} 38^{\prime \prime} 28$ \\
\hline$z_{[\mathrm{C} \mathrm{II}]}$ & $7.5413 \pm 0.0007$ \\
\hline$F_{[\mathrm{C} \mathrm{II]}}\left(\mathrm{Jy} \mathrm{km} \mathrm{s}^{-1}\right)$ & $1.25 \pm 0.17$ \\
\hline $\mathrm{FWHM}_{[\mathrm{C} \mathrm{II}]}\left(\mathrm{km} \mathrm{s}^{-1}\right)$ & $383 \pm 56$ \\
\hline $\mathrm{EW}_{[\mathrm{C} \mathrm{II}]}(\mu \mathrm{m})$ & $1.73 \pm 0.43$ \\
\hline$S_{223.5 \mathrm{GHz}}(\mu \mathrm{Jy})$ & $415 \pm 73$ \\
\hline$S_{135.5 \mathrm{GHz}}(\mu \mathrm{Jy})$ & $<139$ \\
\hline$S_{95 \mathrm{GHz}}(\mu \mathrm{Jy})$ & $<48$ \\
\hline$S_{41 \mathrm{GHz}}(\mu \mathrm{Jy})$ & $15.0 \pm 5.7$ \\
\hline$S_{1.4 \mathrm{GHz}}(\mu \mathrm{Jy})$ & $<432$ \\
\hline$F_{\mathrm{CO}(3-2)}\left(\mathrm{Jy} \mathrm{km} \mathrm{s}^{-1}\right)$ & $<0.081$ \\
\hline$F_{\mathrm{CO}(7-6)}\left(\mathrm{Jy} \mathrm{km} \mathrm{s}^{-1}\right)$ & $<0.13$ \\
\hline$F_{\mathrm{CO}(10-9)}\left(\mathrm{Jy} \mathrm{km} \mathrm{s}^{-1}\right)$ & $<0.32$ \\
\hline$F_{[\mathrm{C} \mathrm{I}]}\left(\mathrm{Jy} \mathrm{km} \mathrm{s}^{-1}\right)$ & $<0.14$ \\
\hline$F_{\mathrm{H}_{2} \mathrm{O}, 1172 \mathrm{GHz}}\left(\mathrm{Jy} \mathrm{km} \mathrm{s}^{-1}\right)$ & $<0.30$ \\
\hline$F_{\mathrm{H}_{2} \mathrm{O}, 1918 \mathrm{GHz}}\left(\mathrm{Jy} \mathrm{km} \mathrm{s}^{-1}\right)$ & $<0.33$ \\
\hline$L_{\mathrm{FIR}}\left(L_{\odot}\right)$ & $(0.5-1.4) \times 10^{12}$ \\
\hline$L_{\mathrm{TIR}}\left(L_{\odot}\right)$ & $(0.8-2.0) \times 10^{12}$ \\
\hline$L_{[\mathrm{C} \mathrm{II]}}\left(L_{\odot}\right)$ & $(1.6 \pm 0.2) \times 10^{9}$ \\
\hline$L_{[\mathrm{C} \text { I] }}\left(L_{\odot}\right)$ & $<7.8 \times 10^{7}$ \\
\hline$L_{\mathrm{CO}(3-2)}^{\prime}\left(\mathrm{K} \mathrm{km} \mathrm{s}^{-1} \mathrm{pc}^{2}\right)$ & $<1.5 \times 10^{10}$ \\
\hline $\operatorname{SFR}_{\mathrm{TIR}}\left(M_{\odot} \mathrm{yr}^{-1}\right)$ & $120-300$ \\
\hline $\operatorname{SFR}_{[\mathrm{C} \mathrm{II}]}\left(M_{\odot} \mathrm{yr}^{-1}\right)$ & $85-545$ \\
\hline$M_{d}\left(M_{\odot}\right)$ & $(0.6-4.3) \times 10^{8}$ \\
\hline$M_{\mathrm{C}^{+}}\left(M_{\odot}\right)$ & $4.9 \times 10^{6}$ \\
\hline$M_{\mathrm{H}_{2}}\left(M_{\odot}\right)$ & $<1.2 \times 10^{10}$ \\
\hline
\end{tabular}

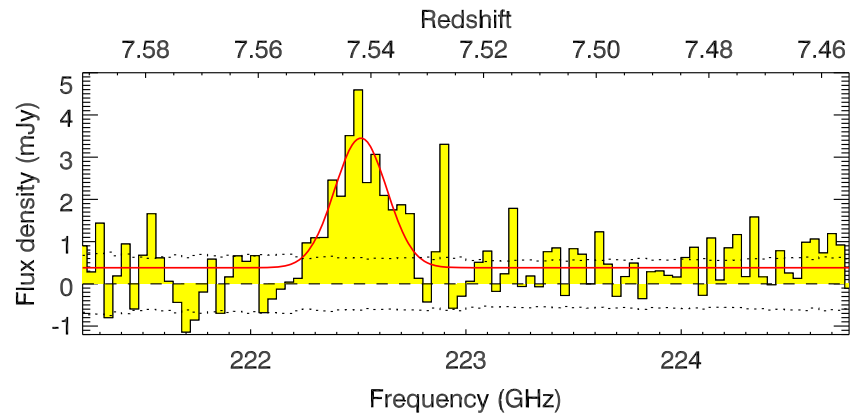

Figure 1. NOEMA spectrum of the redshifted [C II] emission line and the underlying continuum in $\mathrm{J} 1342+092$, extracted from the peak pixel in the datacube. The bin size is $40 \mathrm{MHz}$, which corresponds to $\sim 54 \mathrm{~km} \mathrm{~s}^{-1}$. The dotted lines indicate $+\sigma$ and $-\sigma$, with $\sigma$ being the noise in each bin. The red, solid line is a flat continuum plus Gaussian fit to the spectrum (the fit values are reported in Table 1).

\subsection{Jansky Very Large Array (VLA) Observations}

We searched for $\mathrm{CO}(3-2)$ emission from $\mathrm{J} 1342+0928$ with the VLA in 2017 April. The redshift of the source places the line at $40.4852 \mathrm{GHz}$. The data also provided a deep continuum observation at $41 \mathrm{GHz}$. A total of $9 \mathrm{hr}(7 \mathrm{hr}$ on-source), was spent using the 8 bit, $2 \mathrm{GHz}$ bandwidth correlator mode for highest line sensitivity. An additional $3 \mathrm{hr}$ was spent using 3 bit, $8 \mathrm{GHz}$ bandwidth from 40 to $48 \mathrm{GHz}$ for an additional continuum measurement.

Standard phase and amplitude calibration was performed, using J1331+305 to set the absolute gain scale and bandpass, and $\mathrm{J} 1347+122$ to determine complex gains as a function of time. Phase stability was excellent. 
[CII]

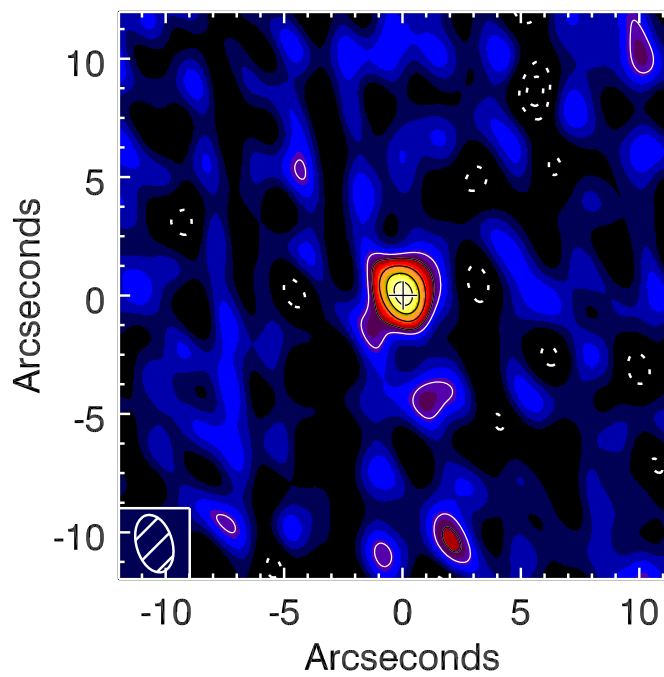

223.5 $\mathrm{GHz}$ continuum
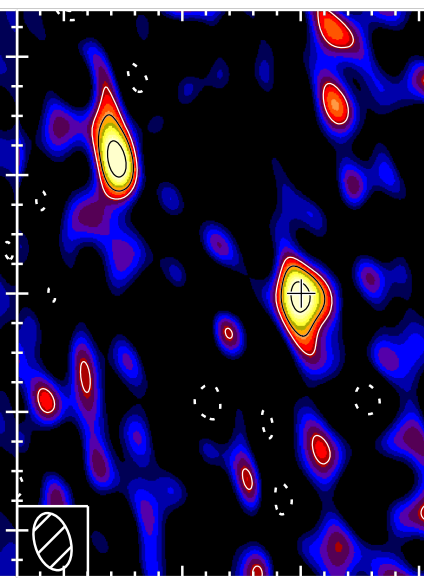
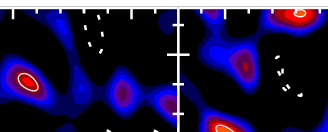

41.0 GHz continuum

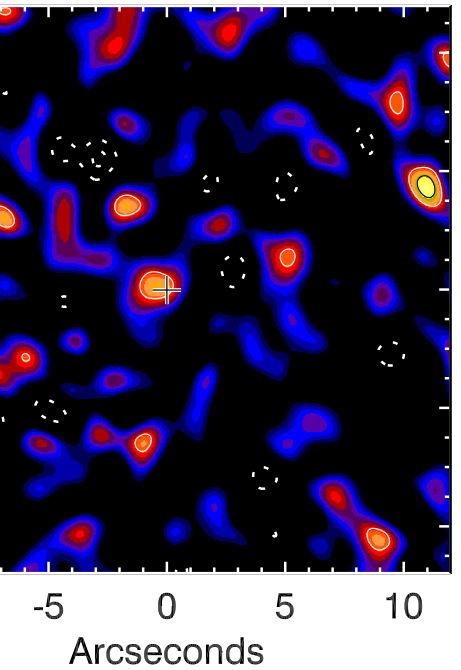

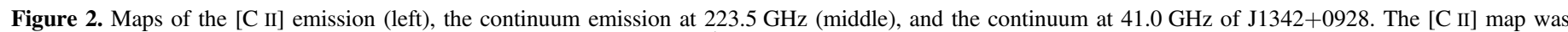

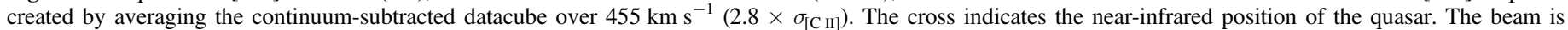

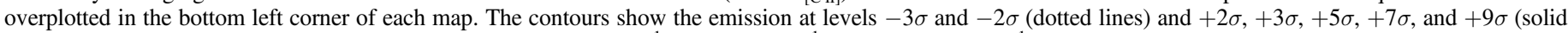

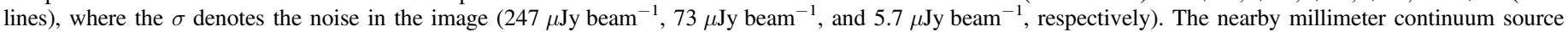
(Section 2.1) can be seen toward the northeast in the middle panel.

The line data were imaged using natural weighting and smoothed to a velocity resolution of $44.5 \mathrm{~km} \mathrm{~s}^{-1}$. The synthesized beam is 2 "! $2 \times 2$ "! 0 , and the rms noise per channel was $0.10 \mathrm{mJy}^{\text {beam }}{ }^{-1}$. We also created a $41.0 \mathrm{GHz}$ continuum image by suitably combining all the data. The rms noise of this continuum image is $5.7 \mu \mathrm{Jy}$ beam $^{-1}$. No line was found, but a potential continuum source is reported (Section 3.1).

\section{The Host Galaxy of $\mathbf{J 1 3 4 2}+0928$ at $z=\mathbf{7 . 5}$}

Our NOEMA observations reveal the gas and dust present in the host galaxy of J1342+0928. In Figure 1, we show the spectrum of the $[\mathrm{C} \mathrm{II}]$ emission line and the underlying dust continuum. A summary of the measurements is given in Table 1.

\subsection{Far-infrared Luminosity and Implied Dust Mass}

The dust continuum around the redshifted [C II] emission (rest-frame wavelength of $\sim 158 \mu \mathrm{m}$ ) has been detected at a signal-to-noise ratio $(\mathrm{S} / \mathrm{N}) \sim 6$ and a strength of $S_{223.5 \mathrm{GHz}}=415 \pm 73 \mu \mathrm{Jy}$ (Figures 1 and 2). The source is not resolved with the 2 !" $4 \times 1$ "' $5\left(12.1 \times 7.3 \mathrm{kpc}^{2}\right)$ beam. We also estimated the source size in the $u v$ plane and derive a source radius $<0$ " 5 , which is consistent with the size measurement of the continuum image. The position of the quasar host, R.A. $=13^{\mathrm{h}} 42^{\mathrm{m}} 08^{\mathrm{s}} .097$; decl. $=+09^{\circ} 28^{\prime} 38^{\prime \prime} \cdot 28$, is consistent with the near-infrared location of the quasar (Bañados et al. 2017). The host galaxy is not detected in continuum in the other NOEMA setups down to $3 \sigma$ continuum limits of $S_{135.5 \mathrm{GHz}}<139 \mu \mathrm{Jy}$ and $S_{95 \mathrm{GHz}}<48 \mu \mathrm{Jy}$. The VLA continuum map shows a potential source $\left(S_{41 \mathrm{GHz}}=\right.$ $15.0 \pm 5.7 \mu \mathrm{Jy}^{\text {beam }^{-1}}$ and $\mathrm{S} / \mathrm{N} \sim 2.6$; Figure 2), located $\sim 0$ !'7 from the [C II] emission of $\mathrm{J} 1342+0928$.

To compute the far-infrared (rest-frame 42.5-122.5 $\mu \mathrm{m}$ ) and total infrared (TIR; $8-1000 \mu \mathrm{m}$ ) luminosities, $L_{\mathrm{FIR}}$ and $L_{\mathrm{TIR}}$, and the dust mass $M_{d}$ in $\mathrm{J} 1342+0928$, we follow the same procedure as outlined in Venemans et al. (2016). In summary, we utilize three different models to estimate dust emission: a modified black body (MBB) with a dust temperature $T_{d}=47 \mathrm{~K}$ and an emissivity index of $\beta=1.6$ (e.g., Beelen et al. 2006) and two templates of local star-forming galaxies (Arp220 and M82) from Silva et al. (1998). We also take the effect of the cosmic microwave background (CMB) on the dust emission into account (e.g., da Cunha et al. 2013; Venemans et al. 2016). The mass of dust is derived both by assuming an opacity index of $\kappa_{\lambda}=0.77(850 \mu \mathrm{m} / \lambda)^{\beta} \mathrm{cm}^{2} \mathrm{~g}^{-1}$ (Dunne et al. 2000) and from scaling the Arp220 and M82 templates (Silva et al. 1998). We stress that due to the unknown shape of the dust continuum, the FIR and TIR luminosities remain highly uncertain, while the SFR and dust mass we derive crucially depend on the applicability of local correlations to this high-redshift source.

Scaling the NOEMA continuum detection of $S_{223.5 \mathrm{GHz}}=$ $415 \pm 73 \mu \mathrm{Jy}$ to the three dust spectral energy distribution (SED) models results in luminosities of $L_{\mathrm{FIR}}=(0.5-1.4) \times$ $10^{12} L_{\odot}$ and $L_{\mathrm{TIR}}=(0.8-2.0) \times 10^{12} L_{\odot}$. The derived dust mass is $M_{d}=(0.6-4.3) \times 10^{8} M_{\odot}$. Applying the local scaling relation between $L_{\mathrm{TIR}}$ and SFR from Murphy et al. (2011) and assuming the infrared luminosity is dominated by star formation (e.g., Leipski et al. 2014) results in an SFR of $120-300 M_{\odot} \mathrm{yr}^{-1}$. This is significantly lower than the SFR derived for some of the quasar hosts at $z \sim 6$ (e.g., Walter et al. 2009), but very similar to the SFR in J1120+0641 at $z=7.1$ (Venemans et al. 2017).

\subsection{Tentative Radio Continuum Emission}

We now look into the origin of the potential VLA continuum detection. The first possibility is that the source is spurious. The $\mathrm{S} / \mathrm{N}$ is only 2.6, and as shown in Figure 2, several positive noise peaks are visible close to the location of the quasar. It is therefore plausible that the $41 \mathrm{GHz}$ detection will disappear when adding more data. On the other hand, if the source is real, then the question is whether it is due to dust emission, free-free emission, or non-thermal processes, e.g., synchrotron radiation. 


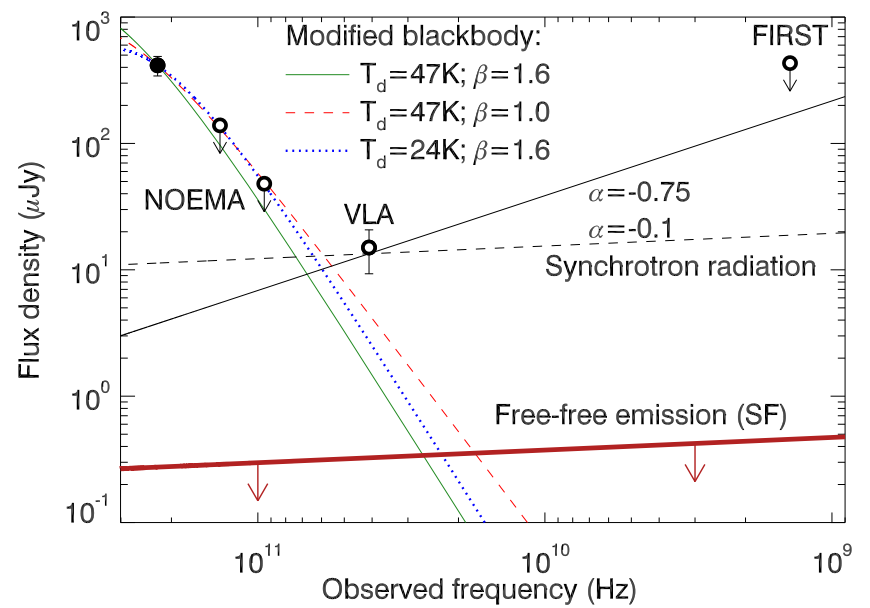

Figure 3. Far-infrared and radio spectral energy distribution of $\mathrm{J} 1342+0928$. The data points from left to right represent the NOEMA 1, 2, and $3 \mathrm{~mm}$ observations, the tentative VLA $41.0 \mathrm{GHz}$ detection, and the FIRST upper limit. Overplotted are three different dust SEDs scaled to the $1 \mathrm{~mm}$ detection and two power laws describing radio synchrotron radiation. The dust model with canonical values $\left(T_{d}=47 \mathrm{~K}\right.$ and $\left.\beta=1.6\right)$ agrees well with the upper limits on the continuum emission at 2 and $3 \mathrm{~mm}$, but predicts a much lower continuum flux density at $41.0 \mathrm{GHz}$. A shallower $\beta(\beta=1.0)$ or a lower dust temperature $\left(T_{d}=24 \mathrm{~K}\right.$, slightly above the CMB temperature at $\left.z=7.54\right)$, illustrated by the dashed and dotted lines, also predicts a $41.0 \mathrm{GHz}$ flux density below that of the tentative VLA source. The upper limit in FIRST does not provide strong constraints on the slope of the radio emission.

The typical quasar dust SED, the MBB with $T_{d}=47 \mathrm{~K}$ and $\beta=1.6$ predicts flux densities of 90,28 , and $1.5 \mu \mathrm{Jy}$ at 135.5 , 95, and $41.0 \mathrm{GHz}$, respectively (Figure 3 ). The limits in the NOEMA $2 \mathrm{~mm}$ and $3 \mathrm{~mm}$ bands are consistent with these expected flux densities, but the flux density measured in the VLA image is significantly $(\sim 10 \times)$ higher than expected from the dust emission. A much shallower emissivity index $(\beta \ll 1.5)$ and/or a lower dust temperature, which would result in a higher flux density at $41 \mathrm{GHz}$, can be ruled out by the nondetections at 135.5 and $95 \mathrm{GHz}$ (Figure 3). Based on the derived SFR in the host galaxy $\left(\mathrm{SFR}=85-545 M_{\odot} \mathrm{yr}^{-1}\right.$; Table 1), the strength of free-free emission at $41.0 \mathrm{GHz}$ is negligible $\left(S_{\mathrm{ff}} \ll 1 \mu \mathrm{Jy}\right.$; e.g., Yun \& Carilli 2002). Alternatively, the flux density could be due to synchrotron radiation. We can estimate the radio loudness of the quasar using the radio-to-optical flux density ratio $R=S_{5 \mathrm{GHz} \text {,rest }} / S_{4400} \AA$,rest with $S_{5 \mathrm{GHz} \text {,rest }}$ and $S_{4400} \AA$,rest the flux densities at rest-frame $5 \mathrm{GHz}$ and $4400 \AA$, respectively (Kellermann et al. 1989). Assuming a radio continuum can be described by a power law $\left(f_{\nu} \propto \nu^{\alpha}\right)$ with $\alpha=-0.75$ (e.g., Bañados et al. 2015), we derive $S_{5 \mathrm{GHz} \text {,rest }}=363 \mu \mathrm{Jy}$. Following Bañados et al. (2015), we derive $S_{4400 \AA \text {,rest }}=29 \mu \mathrm{Jy}$ from the WISE W1 magnitude (W1 = 20.17). We obtain $R=12.4$, making $\mathrm{J} 1342+0928$ a radio-loud quasar (where radio-loud is defined as $R>10$ ). Note that this is still consistent with the nondetection in the FIRST survey, with a $3 \sigma$ upper limit of $S_{1.4 \mathrm{GHz}}<432 \mu \mathrm{Jy}$, as the expected flux density for J1342+0928 is $S_{1.4 \mathrm{GHz}} \approx 190 \mu \mathrm{Jy}$ (Figure 3 ). Deeper imaging at radio frequencies will provide a definitive answer.

\section{3. [C II] Luminosity}

We detect the [C II] emission line in $\mathrm{J} 1342+0928$ in the continuum-subtracted $[\mathrm{C} \mathrm{II}]$ map (Figure 2 ) with an $\mathrm{S} / \mathrm{N} \sim 10$. The spectrum, extracted from the peak pixel in the datacube, is shown in Figure 1. From a Gaussian fit to the line, we derive a

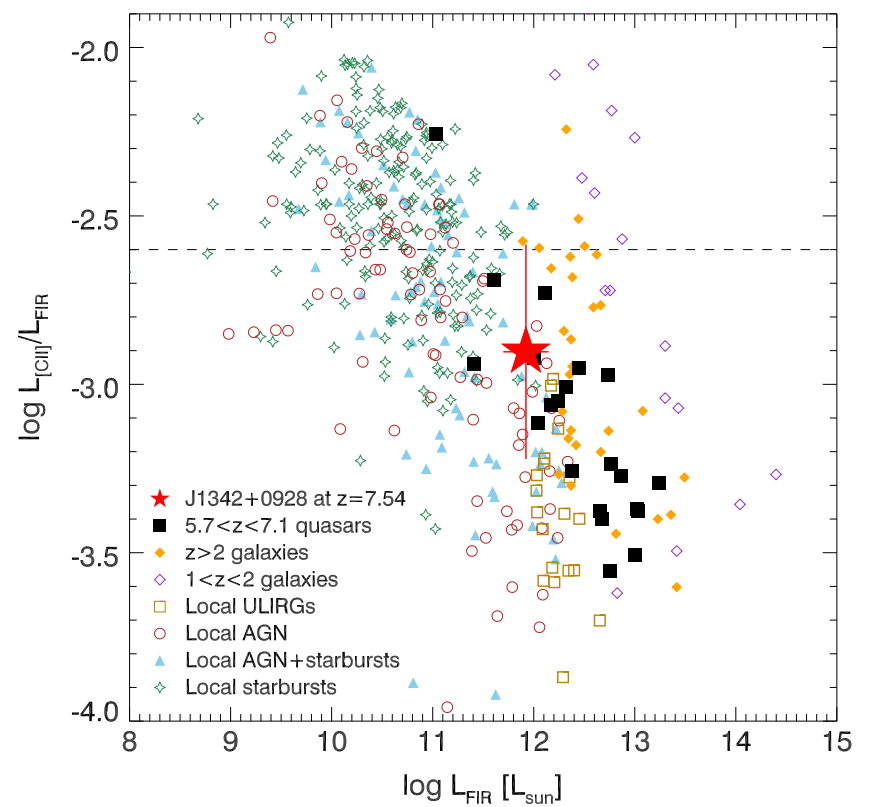

Figure 4. [C II]-to-FIR luminosity ratio vs. FIR luminosity. Plotted are values for starburst galaxies and active galactic nuclei (AGNs) in the local universe and at high redshift and for local ULIRGs (Maiolino et al. 2005; Walter et al 2009; Díaz-Santos et al. 2013; Venemans et al. 2016; Mazzucchelli et al. 2017 and references therein). The value for $\mathrm{J} 1342+0928$ is plotted as a red star. The dashed line indicates the median $L_{[\mathrm{C} \mathrm{II}]} / L_{\mathrm{FIR}}$ ratio of local star-forming galaxies (Díaz-Santos et al. 2013).

redshift of $z_{[\mathrm{C} \mathrm{II}]}=7.5413 \pm 0.0007$, a line flux of $F_{[\mathrm{C} \mathrm{II}]}=$ $1.25 \pm 0.17 \mathrm{Jy} \mathrm{km} \mathrm{s}^{-1}$, and a dispersion of $\sigma_{[\mathrm{C} \mathrm{III}]}=163 \pm$ $24 \mathrm{~km} \mathrm{~s}^{-1}\left(\mathrm{FWHM}_{[\mathrm{C} \text { II] }}=383 \pm 56 \mathrm{~km} \mathrm{~s}^{-1}\right)$; see Table 1. This corresponds to a [C II] luminosity in this quasar of $L_{[\mathrm{C} \text { II] }}=$ $(1.6 \pm 0.2) \times 10^{9} L_{\odot}$, which is roughly $\sim 15 \%$ brighter than $\mathrm{J} 1120+0641$ at $z=7.1$ (Venemans et al. 2017) and a factor 3-5 fainter than the most [C II] luminous quasar at $z \sim 6$ (e.g., Maiolino et al. 2005; Wang et al. 2013).

The redshift derived from the [C II] line is higher than that derived from the UV emission lines of the quasar. The C IV and $\mathrm{Mg}$ II lines are blueshifted by $6580 \pm 270 \mathrm{~km} \mathrm{~s}^{-1}$ and $500 \pm 140 \mathrm{~km} \mathrm{~s}^{-1}$ with respect to the [C II] line. The Mg II shift is close to the mean blueshift of the $\mathrm{Mg}$ II line of $480 \mathrm{~km} \mathrm{~s}^{-1}$ found in a sample of $z \sim 6-7$ quasars (e.g., Venemans et al. 2016). This could indicate the presence of an outflow (e.g., Mazzucchelli et al. 2017).

We measure a rest-frame $[\mathrm{C} \mathrm{II}]$ equivalent width of $\mathrm{EW}_{[\mathrm{C} \mathrm{II}}=$ $1.73 \pm 0.43 \mu \mathrm{m}$, which is consistent with the mean $\mathrm{EW}_{[\mathrm{C} I \mathrm{II}}$ of local starburst galaxies (which have $\left\langle\mathrm{EW}_{[\mathrm{C}}\right.$ II $\left._{\mathrm{I}}\right\rangle=1.27 \pm$ $0.53 \mu \mathrm{m}$; see e.g., Díaz-Santos et al. 2013; Sargsyan et al. 2014) and higher than those of luminous $\left(M_{1450}<-27\right)$ quasars at $z \sim 6$ (e.g., Wang et al. 2013). The [C II]-to-FIR luminosity ratio is $L_{[\mathrm{C}}$ II] $/ L_{\mathrm{FIR}}=(0.6-2.6) \times 10^{-3}$ (Figure 4$)$, again consistent within the large uncertainties with the $L_{\text {[C II] }} / L_{\mathrm{FIR}}$ ratio of local star-forming galaxies that have a median $L_{[\mathrm{C} \text { II] }} / L_{\mathrm{FIR}}=2.5 \times 10^{-3}$ (e.g., Díaz-Santos et al. 2013).

We can estimate the SFR from the [C II] emission using the SFR- $L_{[\mathrm{C} \text { II] }}$ relations for high-redshift $(z>0.5)$ galaxies of De Looze et al. (2014):

$$
\mathrm{SFR}_{[\mathrm{C} \text { II }]} / M_{\odot} \mathrm{yr}^{-1}=3.0 \times 10^{-9}\left(L_{[\mathrm{C} \mathrm{II}]} / L_{\odot}\right)^{1.18},
$$

with a systematic uncertainty of a factor of $\sim 2.5$. With $L_{[\mathrm{C} \mathrm{II}]}=$ $(1.6 \pm 0.2) \times 10^{9} L_{\odot}$ we derive $\mathrm{SFR}_{[\mathrm{C} \text { II }}=85-545 M_{\odot} \mathrm{yr}^{-1}$, 
which is similar to the SFR based on the TIR luminosity (Section 3.1).

The [C II] emission is not resolved in the 2 ." $5 \times 1$ ". 5 beam (Figure 2). We fitted a 2D Gaussian to the [C II] map using the CASA task "imfit" and we derive a $1 \sigma$ upper limit on the size of 1 !' $7 \times 1$ !" 2 (FWHM). A similar limit on the source diameter of $D<1$ "' 0 is found when fitting a 1D Gaussian to the $u v$ data. This translates to an upper limit on the size of the [C II]emitting region of $8.4 \times 5.9 \mathrm{kpc}^{2}$ or a diameter of $D \lesssim 7 \mathrm{kpc}$. Approved observations with the Atacama Large Millimeter/ submillimeter Array (ALMA) at higher spatial resolution will put tighter constraints on the size of the host galaxy.

From the strength of the [C II] emission line, we can derive the mass of singly ionized carbon. In analogy to the formula to compute the mass of neutral carbon provided in Weiß et al. (2005) and assuming optically thin [C II] emission, the mass of singly ionized carbon can be calculated using

$$
\begin{aligned}
M_{\mathrm{C}^{+}} / M_{\odot} & \left.=C m_{\mathrm{C}} \frac{8 \pi k \nu_{0}^{2}}{h c^{3} A} Q\left(T_{\mathrm{ex}}\right) \frac{1}{4} e^{91.2 / T_{\mathrm{ex}}} L_{[\mathrm{C}}^{\prime} \mathrm{II}\right] \\
& =2.92 \times 10^{-4} Q\left(T_{\mathrm{ex}}\right) \frac{1}{4} \mathrm{e}^{91.2 / T_{\mathrm{ex}}} L_{[\mathrm{C} \text { II] }}^{\prime},
\end{aligned}
$$

with $C$ the conversion between $\mathrm{pc}^{2}$ and $\mathrm{cm}^{2}, m_{\mathrm{C}}$ the mass of a carbon atom, $A=2.29 \times 10^{-6} \mathrm{~s}^{-1}$ the Einstein coefficient (Nussbaumer \& Storey 1981), $Q\left(T_{\text {ex }}\right)=2+4 \mathrm{e}^{-91.2 / T_{\text {ex }}}$ the C II partition function, and $T_{\mathrm{ex}}$ the excitation temperature. As [C II] is emitted from the outer layers of photon-dominated region (PDR) clouds, $T_{\mathrm{ex}} \gtrsim 100 \mathrm{~K}$ is a good assumption (see, e.g., Meijerink et al. 2007). Setting $T_{\mathrm{ex}}=100 \mathrm{~K}$ we derive $M_{\mathrm{C}^{+}}=4.9 \times 10^{6} M_{\odot}$. For $T_{\mathrm{ex}}=200 \mathrm{~K}(75 \mathrm{~K})$, the mass would be $\sim 20 \%$ lower (higher).

\subsection{Limits on the $\mathrm{CO}$ and $[\mathrm{CI}$ / Luminosity}

We do not detect any of the other targeted emission lines in J1342+0928. To derive upper limits on the line fluxes, we averaged the datacubes over $2.8 \times \sigma_{[\mathrm{C} \mathrm{II}]}\left(460 \mathrm{~km} \mathrm{~s}^{-1}\right)$. We measured the following $3 \sigma$ upper limits: $F_{\mathrm{CO}(10-9)}<0.32$ $\mathrm{Jy} \mathrm{km} \mathrm{s}{ }^{-1}, \quad F_{\mathrm{CO}(7-6)}<0.13 \mathrm{Jy} \mathrm{km} \mathrm{s}^{-1}, \quad F_{[\mathrm{C} \mathrm{I}]}<0.14 \mathrm{Jy} \mathrm{km} \mathrm{s}^{-1}$, $F_{\mathrm{CO}(3-2)}<0.081 \mathrm{Jy} \mathrm{km} \mathrm{s}^{-1}$, and $F_{\mathrm{H}_{2} \mathrm{O}, 1172 \mathrm{GHz}}<0.30 \mathrm{Jy} \mathrm{km} \mathrm{s}^{-1}$.

The limits on the $\mathrm{CO}$ luminosity are $L_{\mathrm{CO}(10-9)}^{\prime}<5.2 \times$ $10^{9} \mathrm{~K} \mathrm{~km} \mathrm{~s}^{-1} \mathrm{pc}^{2}, \quad L_{\mathrm{CO}(7-6)}^{\prime}<4.3 \times 10^{9} \mathrm{~K} \mathrm{~km} \mathrm{~s}^{-1} \mathrm{pc}^{2}, \quad$ and $L_{\mathrm{CO}(3-2)}^{\prime}<1.5 \times 10^{10} \mathrm{~K} \mathrm{~km} \mathrm{~s}^{-1} \mathrm{pc}^{2}$. We can estimate a limit on the molecular gas mass $M_{\mathrm{H}_{2}}$ by utilizing $M_{\mathrm{H}_{2}}=\alpha L_{\mathrm{CO}(1-0)}^{\prime}$ with $\alpha$ the $\mathrm{CO}$ luminosity-to-gas mass conversion factor. Assuming the $\mathrm{CO}(3-2)$ emission is thermalized (e.g., Riechers et al. 2009), the $\mathrm{CO}(1-0)$ luminosity is given by $L_{\mathrm{CO}(1-0)}^{\prime}=L_{\mathrm{CO}(3-2)}^{\prime}$. Adopting $\alpha=0.8$ (e.g., Downes \& Solomon 1998), we set an upper limit on the molecular gas mass of $M_{\mathrm{H}_{2}}<1.2 \times 10^{10} M_{\odot}$.

The limiting luminosity of the [C I] line is $L_{[\mathrm{C} \mathrm{I}]}<7.8 \times$ $10^{7} L_{\odot}$. With a measured [C II] luminosity of $L_{[\mathrm{C} \mathrm{II}]}=(1.6 \pm$ $0.2) \times 10^{9} L_{\odot}$ (Section 3.3), we can set a lower limit to the [C II]-to-[C I] luminosity ratio of $L_{[\mathrm{C} \text { II] }} / L_{[\mathrm{C} \text { I] }}>18$. Following Venemans et al. (2017), we can compare this luminosity ratio to those predicted by the ISM models of Meijerink et al. (2007). From the measured luminosity ratio we can exclude that the line emission originates from a region where the X-ray radiation from the accreting black hole is dominating the emission.

\subsection{Dynamical Mass Estimate}

From the velocity dispersion $\sigma$ of the [C II] emission and the radius $R$ of the line emitting region, we can estimate a dynamical mass of the quasar host galaxy by utilizing the virial theorem: $M_{\text {dyn }}=3 R \sigma^{2} / 2 G$ with $G$ as the gravitational constant. Assuming that the velocity dispersion can be derived from the Gaussian fit to the [C II] emission (Figure 1), and adopting a maximum radius of the [C II] emission of $R<3.5 \mathrm{kpc}$ (Section 3.3), we infer a dynamical mass $M_{\text {dyn }}<3.2 \times 10^{10} M_{\odot}$. If instead we assume that the $[\mathrm{CII}]$ emission is in a rotating disk with inclination angle $i$ (e.g., Wang et al. 2013; Willott et al. 2015; Venemans et al. 2016), we derive a higher dynamical mass of $M_{\mathrm{dyn}}<1.0 \times 10^{11} / \sin ^{2}(i) M_{\odot}$. Adopting $i=55^{\circ}$, the median inclination angle of $z \sim 6$ quasar hosts (Wang et al. 2013), the dynamical mass of J1342+0928 becomes $M_{\text {dyn }}<1.5 \times$ $10^{11} M_{\odot}$, which is $\lesssim 190 \times$ higher that of the black hole (Bañados et al. 2017). To more accurately constrain the dynamical mass, high spatially resolved observations of the $[\mathrm{C} \mathrm{II}]$ emission are necessary.

\section{Concluding Remarks}

We presented the detection of copious amounts of dust $\left(\sim 10^{8} M_{\odot}\right)$ and metal-enriched gas $\left(\sim 5 \times 10^{6} M_{\odot}\right.$ of carbon in the singly ionized phase only) in a quasar host galaxy $690 \mathrm{Myr}$ after the Big Bang. The enrichment of the ISM in this source appears similar to other quasars at $z=6-7$ (e.g., Riechers et al. 2009; Wang et al. 2013; Venemans et al. 2016) but much higher than what is typically found in non-quasar host galaxies at these redshifts (e.g., Watson et al. 2015; Pentericci et al. 2016).

We can only speculate which mechanism is responsible for the high mass in metals so early after the Big Bang. Because of the young cosmic age, asymptotic giant branch stars are thought to play only a marginal role (e.g., Morgan \& Edmunds 2003; Juarez et al. 2009; Gall et al. 2011a). On the other hand, type II supernovae ( $\mathrm{SNe}$ ) can produce significant amounts of dust, up to $\sim 1 M_{\odot}$ per $\mathrm{SN}$ (e.g., Matsuura et al. 2015). For an initial mass function (IMF) similar to that of the Milky Way, the number of SNe is 1 per $200 M_{\odot}$ of stars formed (e.g., Diehl et al. 2006). The implied stellar mass of $\mathrm{J} 1342+0928$ would then be $M_{*}=2 \times 10^{10} M_{\odot}$. Assuming a top-heavy IMF the implied stellar mass would be reduced by a factor of $\sim 3$. In either case, such a massive stellar population should be easily detectable with the combined sensitivity, resolution, and wavelength coverage of the James Webb Space Telescope (JWST).

At these extreme redshifts, population III stars also provide a plausible enrichment mechanism. Metal-free stars with a mass $140<M / M_{\odot}<260$ could have dust yield as high as 15\%-30\% (e.g., Nozawa et al. 2003). Neglecting dust destruction, one would "only" require 2 million population III stars of $200 M_{\odot}$ to create a mass of $10^{8} M_{\odot}$ in dust, although the fast metal pollution may prevent the formation of so many population III stars (e.g., Maio et al. 2010).

The presented observations showcase how the study of quasar host galaxies at the highest redshifts can shed new light on the dawn of galaxy formation. Future ALMA and JWST observations will allow us to constrain the molecular gas mass, determine the shape of the FIR dust emission, and measure the 
size of the gas reservoir and to reveal the stellar population in the quasar host of this system.

We thank the referee for providing valuable comments and suggestions. B.P.V., F.W., and E.P.F. acknowledge funding through the ERC grant "Cosmic Dawn." Support for R.D. was provided by the DFG priority program 1573 "The physics of the interstellar medium." We thank Amanda Karakas for help with estimating the metal production in stars. We are grateful to the JVLA and NOEMA for providing DDT observations. This work is based on observations carried our under project number E16AH with the IRAM NOEMA Interferometer. IRAM is supported by INSU/CNRS (France), MPG (Germany), and IGN (Spain).

Facilities: IRAM:Interferometer, EVLA.

\section{ORCID iDs}

Bram P. Venemans (iD https://orcid.org/0000-0001-9024-8322 Fabian Walter (iD https://orcid.org/0000-0003-4793-7880 Roberto Decarli (i) https://orcid.org/0000-0002-2662-8803 Eduardo Bañados (1D https://orcid.org/0000-0002-2931-7824 Chris Carilli (iD https://orcid.org/0000-0001-6647-3861 Elisabete da Cunha (1) https://orcid.org/0000-0001-9759-4797 Xiaohui Fan (1) https://orcid.org/0000-0003-3310-0131 Emanuele Paolo Farina (iD https://orcid.org/0000-00026822-2254

Chiara Mazzucchelli (iD https:// orcid.org/0000-0002-

5941-5214

Hans-Walter Rix (iD https://orcid.org/0000-0003-4996-9069

Axel Weiss (iD) https://orcid.org/0000-0003-4678-3939

\section{References}

Agarwal, B., Khochfar, S., Johnson, J. L., et al. 2012, MNRAS, 425, 2854 Bañados, E., Venemans, B. P., Decarli, R., et al. 2016, ApJS, 227, 11 Bañados, E., Venemans, B. P., Mazzucchelli, C., et al. 2017, Natur, https:// doi.org/10.1038/nature25180

Bañados, E., Venemans, B. P., Morganson, E., et al. 2015, ApJ, 804, 118

Beelen, A., Cox, P., Benford, D. J., et al. 2006, ApJ, 642, 694

Bertoldi, F., Cox, P., Neri, R., et al. 2003, A\&A, 409, L47

da Cunha, E., Groves, B., Walter, F., et al. 2013, ApJ, 766, 13
Decarli, R., Walter, F., Yang, Y., et al. 2012, ApJ, 756, 150

De Looze, I., Cormier, D., Lebouteiller, V., et al. 2014, A\&A, 568, A62

De Rosa, G., Venemans, B. P., Decarli, R., et al. 2014, ApJ, 790, 145

Díaz-Santos, T., Armus, L., Charmandaris, V., et al. 2013, ApJ, 774, 68

Diehl, R., Halloin, H., Kretschmer, K., et al. 2006, Natur, 439, 45

Dietrich, M., Hamann, F., Shields, J. C., et al. 2003, ApJ, 589, 722

Downes, D., \& Solomon, P. M. 1998, ApJ, 507, 615

Dunne, L., Eales, S., Edmunds, M., et al. 2000, MNRAS, 315, 115

Fan, X., Strauss, M. A., Richards, G. T., et al. 2006, AJ, 131, 1203

Ferland, G. 2004, in ASP Conf. Ser. 311, AGN Physics with the Sloan Digital

Sky Survey, ed. G. T. Richards \& P. B. Hall (San Francisco, CA: ASP), 161

Gall, C., Andersen, A. C., \& Hjorth, J. 2011a, A\&A, 528, A14

Gall, C., Hjorth, J., \& Andersen, A. C. 2011b, A\&ARv, 19, 43

Jiang, L., Fan, X., Vestergaard, M., et al. 2007, AJ, 134, 1150

Juarez, Y., Maiolino, R., Mujica, R., et al. 2009, A\&A, 494, L25

Kellermann, K. I., Sramek, R., Schmidt, M., Shaffer, D. B., \& Green, R. 1989 AJ, 98, 1195

Leipski, C., Meisenheimer, K., Walter, F., et al. 2014, ApJ, 785, 154

Maio, U., Ciardi, B., Dolag, K., Tornatore, L., \& Khochfar, S. 2010, MNRAS, 407, 1003

Maiolino, R., Cox, P., Caselli, P., et al. 2005, A\&A, 440, L51

Matsuura, M., Dwek, E., Barlow, M. J., et al. 2015, ApJ, 800, 50

Mazzucchelli, C., Bañados, E., Venemans, B. P., et al. 2017, ApJ, 849, 91

Meijerink, R., Spaans, M., \& Israel, F. P. 2007, A\&A, 461, 793

Michałowski, M. J., Murphy, E. J., Hjorth, J., et al. 2010, A\&A, 522, A15

Morgan, H. L., \& Edmunds, M. G. 2003, MNRAS, 343, 427

Mortlock, D. J., Warren, S. J., Venemans, B. P., et al. 2011, Natur, 474, 616

Murphy, E. J., Condon, J. J., Schinnerer, E., et al. 2011, ApJ, 737, 67

Nozawa, T., Kozasa, T., Umeda, H., Maeda, K., \& Nomoto, K. 2003, ApJ, 598,785

Nussbaumer, H., \& Storey, P. J. 1981, A\&A, 96, 91

Pentericci, L., Carniani, S., Castellano, M., et al. 2016, ApJL, 829, L11

Regan, J. A., Visbal, E., Wise, J. H., et al. 2017, NatAs, 1, 0075

Riechers, D. A., Walter, F., Bertoldi, F., et al. 2009, ApJ, 703, 1338

Sargsyan, L., Samsonyan, A., Lebouteiller, V., et al. 2014, ApJ, 790, 15

Silva, L., Granato, G. L., Bressan, A., \& Danese, L. 1998, ApJ, 509, 103

Venemans, B. P., Findlay, J. R., Sutherland, W. J., et al. 2013, ApJ, 779, 24

Venemans, B. P., McMahon, R. G., Walter, F., et al. 2012, ApJL, 751, L25

Venemans, B. P., Walter, F., Decarli, R., et al. 2017, ApJ, 837, 146

Venemans, B. P., Walter, F., Zschaechner, L., et al. 2016, ApJ, 816, 37

Volonteri, M. 2012, Sci, 337, 544

Walter, F., Bertoldi, F., Carilli, C., et al. 2003, Natur, 424, 406

Walter, F., Riechers, D., Cox, P., et al. 2009, Natur, 457, 699

Wang, R., Wagg, J., Carilli, C. L., et al. 2013, ApJ, 773, 44

Watson, D., Christensen, L., Knudsen, K. K., et al. 2015, Natur, 519, 327

Weiß, A., Downes, D., Henkel, C., \& Walter, F. 2005, A\&A, 429, L25

Willott, C. J., Bergeron, J., \& Omont, A. 2015, ApJ, 801, 123

Yun, M. S., \& Carilli, C. L. 2002, ApJ, 568, 88 